\title{
A method for solving nonlinear Volterra's population growth model of noninteger order
}

\author{
D Baleanu ${ }^{1,2^{*}}$, B Agheli $^{3}$, M Adabitabar Firozja $^{3}$ and M Mohamed Al Qurashi ${ }^{4}$
}

\author{
"Correspondence: \\ dumitru@cankaya.edu.tr \\ 'Department of Mathematics, \\ Çankaya University, Ankara, Turkey \\ Full list of author information is \\ available at the end of the article
}

\begin{abstract}
Many numerical methods have been developed for nonlinear fractional integro-differential Volterra's population model (FVPG). In these methods, to approximate a function on a particular interval, only a restricted number of points have been employed. In this research, we show that it is possible to use the fuzzy transform method (F-transform) to tackle with FVPG. It makes the F-transform preferable to other methods since it can make full use of all points on this interval. We also make a comparison showing that this method is less computational and is more convenient to be utilized for coping with nonlinear integro-differential equation (IDEs), fractional nonlinear integro-differential equation (FIDEs), and fractional ordinary differential equations (FODEs).
\end{abstract}

MSC: $45 \mathrm{~J} 05 ; 26 \mathrm{~A} 33 ; 26 \mathrm{~A} 33$

Keywords: population growth; fuzzy transform; Caputo derivative; integro-differential equation

\section{Introduction}

Fractional arithmetic and fractional differential equations appear in many sciences, including medicine [1], economics [2], dynamical problems [3, 4], chemistry [5], mathematical physics [6], traffic model [7], fluid flow [8], and so on. Scholars and researchers are invited to check books that have been written to take advantage of fractional arithmetic [9-11].

In this research work, we have for the first time shown that it is possible to use the $F$ transform method (FTM) to tackle with the nonlinear Volterra population growth model of noninteger order (FVPG). We have investigated the following FVPG:

$$
D_{t}^{\beta} P(\widetilde{t})=A P(\widetilde{t})-B P^{2}(\widetilde{t})-C P(\widetilde{t}) I_{0}^{\widetilde{t}} P(s), \quad 0<\beta \leq 1, P(0)=P_{0},
$$

where

- $D^{\beta}$ denotes the fractional differential operator of order $\beta$ defined by

$$
D^{\beta} P(t)=\frac{1}{\Gamma(k-\beta)} \int_{0}^{t}(t-s)^{\beta-1} P^{(k)}(s) d s, \quad k-1<\beta \leq k, k \in \mathbb{N},
$$

(c) The Author(s) 2017. This article is distributed under the terms of the Creative Commons Attribution 4.0 International License (http://creativecommons.org/licenses/by/4.0/), which permits unrestricted use, distribution, and reproduction in any medium, provided you give appropriate credit to the original author(s) and the source, provide a link to the Creative Commons license, and indicate if changes were made. 
- the positive parameter $A$ stands for the coefficient of birth rate,

- the positive parameter $B$ is the coefficient of crowding,

- the positive parameter $C$ is the coefficient of toxicity, which demonstrates the important treatment of the population evolution as long as its level falls to zero in the long run,

- $P_{0}$ is the primary population,

- $P=P(\widetilde{t})$ denotes the population at time $\widetilde{t}$, and

- the integral $I_{0}^{\tilde{t}} P(s)=\int_{0}^{\widetilde{s}} P(s) d s$ indicates the effect of toxin gathering.

We utilize the scale population and time by putting $u=\frac{B P}{A}$ and $t=\frac{C \widetilde{C}}{B}$ to gain the following problem:

$$
\left\{\begin{array}{l}
\lambda D^{\beta} u(t)=u(t)-u^{2}(t)-u(t) I_{0}^{t} u(s), \quad 0<\beta \leq 1, \\
u(0)=u_{0} .
\end{array}\right.
$$

In the nondimensional problem (1.3):

- $u(t)$ at time $t$ is the scaled population of similar individuals,

- $\lambda=\frac{C}{A B}$ is a prescribed nondimensional parameter.

The analytical solution for $\beta=1$ of (1.3) is [12]

$$
u(t)=u_{0} \exp \left(\frac{1}{\lambda} \int_{0}^{t}\left(1-u(s)-\int_{0}^{s} u(x) d x\right) d s\right)
$$

which shows that if $u_{0}>0$, then $u(t)$ is positive for all $t$.

Various approximate methods for approximating the VPG and FVPG have been investigated by scholars: Hicdurmaz and Can [13], the pseudospectral method of the Legendre functions of noninteger order; Parand and Delkhosh [14], the generalized Chebyshev orthogonal functions of non-integer order of the first kind and the collocation method; Maleki and Maleki [15], the multidomain Legendre-Gauss pseudospectral approach; Suat Erturk et al. [16], the Padé approximations and differential transform method; Khan et al. [17], a new homotopy perturbation method; Krishnaveni et al. [18], the shifted Legendre polynomial method, Dehghan and Shahini [19], the rational pseudospectral approximation, Ghasemi [20], a new homotopy analysis method; Yildirim and Gulkanat [21], the homotopy-Padé technique; Fathizadeh [22], the hybrid rational Haar wavelets; and so on.

The $F$-transform has been implemented for dealing with ordinary differential equations as compared with many other classical procedures [23]. In approximate methods, for the purpose of approximating a function on particular interval, only a restricted number of points are used. The $F$-transform is preferable to other methods because it uses all points in this interval.

The FTM has recently been utilized in [24-26] to find an approximate solution of the first-order fuzzy differential equations and two-point boundary value problems.

Along the same line of research, Chen and Shen [27] have established an algorithm to gain the numerical solutions of second-order primary amount problems.

\section{Fuzzy partition and fuzzy transform}

In this section, we outline the main definitions of the $F$-transform to be utilized in the subsequent sections of numerical implementations. 
Definition 2.1 ([23]) Presuming that for $n \geq 2, t_{1}=a<t_{2}<\cdots<t_{n-1}<t_{n}=b$ are specified nodes, we express that fuzzy sets $B_{1}, \ldots, B_{n}$ defined on $[a, b]$ with their membership functions $B_{1}(t), \ldots, B_{n}(t)$ form a fuzzy partition of $[a, b]$ if they satisfy the following conditions:

(1) the functions $B_{k}$ on $[a, b]$ taking values in $[0,1]$ are continuous, $\sum_{k=1}^{n} B_{k}(t)=1$ for $t \in[a, b]$, and $B_{k}\left(t_{k}\right)=1$,

(2) $B_{k}(t)=0$ if $t \notin\left(t_{k-1}, t_{k+1}\right)$ with $t_{0}=a$ and $t_{n+1}=b$,

(3) $B_{k}(t)$ increases on $\left[t_{k-1}, t_{k}\right]$ for $k=2, \ldots, n$ and decreases on $\left[t_{k}, t_{k+1}\right], k=1, \ldots, n-1$.

The membership functions $B_{1}, B_{2}, \ldots, B_{n}$ are called basic functions (BFs).

The following formulas give standard triangular membership functions:

$$
\begin{aligned}
& B_{1}(t)= \begin{cases}1-\frac{t-t_{1}}{h_{1}}, & t_{1} \leq t \leq t_{2}, \\
0 & \text { otherwise, }\end{cases} \\
& B_{k}(t)= \begin{cases}\frac{t-t_{k-1}}{h_{k-1}}, & t_{k-1} \leq t \leq t_{k}, \\
1-\frac{t-t_{k}}{h_{k}}, & t_{k} \leq t \leq t_{k+1}, \\
0 & \text { otherwise, }\end{cases} \\
& B_{n}(t)= \begin{cases}\frac{t-t_{n-1}}{h_{n-1}}, & t_{n-1} \leq t \leq t_{n}, \\
0 & \text { otherwise. }\end{cases}
\end{aligned}
$$

The following formulas for $k=2, \ldots, n-1$ give standard sinusoidal membership functions:

$$
\begin{aligned}
& B_{1}(t)= \begin{cases}0.5\left(1+\cos \frac{\pi}{h}\left(t-t_{1}\right)\right), & t_{1} \leq t \leq t_{2}, \\
0 & \text { otherwise, }\end{cases} \\
& B_{k}(t)= \begin{cases}0.5\left(1+\cos \frac{\pi}{h}\left(t-t_{k}\right)\right), & t_{k-1} \leq t \leq t_{k+1}, \\
0 & \text { otherwise, }\end{cases} \\
& B_{n}(t)= \begin{cases}0.5\left(1+\cos \frac{\pi}{h}\left(t-t_{n}\right)\right), & t_{n-1} \leq t \leq t_{n}, \\
0 & \text { otherwise, }\end{cases}
\end{aligned}
$$

where $h_{k}=t_{k+1}-t_{k}, k=1, \ldots, n-1$. A fuzzy partition of $[a, b]$ for $k=1,2, \ldots, n-1$ is uniform if $t_{k+1}-t_{k}=h=\frac{b-a}{n-1}$ and two additional properties are satisfied:

(4) $B_{k}\left(t_{k}-t\right)=B_{k}\left(t_{k}+t\right)$ for all $t \in[0, h], k=2, \ldots, n-1$,

(5) $B_{k}(t)=B_{k-1}(t-h)$ and $B_{k+1}(t)=B_{k}(t-h)$ for all $k=2, \ldots, n-1$ and $t \in\left[t_{k}, t_{k+1}\right]$.

Lemma 2.2 ([23]) Let $n \geq 3$, and let $B_{1}, B_{2}, \ldots, B_{n}$ be the BFs forming a uniform partition of $[a, b]$. Then

$$
\int_{t_{1}}^{t_{2}} B_{1}(t) d t=\int_{t_{n-1}}^{t_{n}} B_{n}(t) d t=\frac{h}{2}
$$

and

$$
\int_{t_{k-1}}^{t_{k+1}} B_{k}(t) d t=h
$$

for $k=2, \ldots, n-1$, where $h$ is the distance between two neighboring nodes. 
Lemma 2.3 Let $B_{1}, B_{2}, \ldots, B_{n}, n \geq 3$, be the sinusoidal membership functions for BFs forming a uniform partition of $[a, b]$. Then

$$
\int_{0}^{t_{k}} B_{i}(t) d t= \begin{cases}h, & k \geq i+1 \\ \frac{h}{2}, & k=i \\ 0, & k \leq i-1\end{cases}
$$

Proof Regarding Definition 2.1, if $t \notin\left(t_{i-1}, t_{i+1}\right)$, then $B_{i}(t)=0$. Otherwise, if $k \leq i-1$, then $t \leq t_{k}$, so $B_{i}(t)=0$, and we have $\int_{0}^{t_{k}} B_{i}(t) d t=0$.

By Lemma 2.2 and Definition 2.1, if $k=i$, then

$$
\int_{0}^{t_{k}} B_{i}(t) d t=\int_{0}^{t_{k-1}} B_{i}(t) d t+\int_{t_{k-1}}^{t_{k}} B_{i}(t) d t=0+\frac{h}{2}=\frac{h}{2} .
$$

If $k \geq i+1$, then, by Lemma 2.2 and Definition 2.1,

$$
\int_{0}^{t_{k}} B_{i}(t) d t=\int_{0}^{t_{k-1}} B_{i}(t) d t+\int_{t_{k-1}}^{t_{k+1}} B_{i}(t) d t+\int_{t_{k+1}}^{t_{k}} B_{i}(t) d t=h .
$$

Definition 2.4 ([23]) Let $f$ be any function from $C([a, b])$, and let $B_{1}, B_{2}, \ldots, B_{n}$ be BFs that form a fuzzy partition of $[a, b]$. We claim that the $n$-tuple $\left[F_{1}, F_{2}, \ldots, F_{n}\right]$ of real numbers given by

$$
F_{k}=\frac{\int_{a}^{b} f(t) B_{k}(t) d t}{\int_{a}^{b} B_{k}(t) d t}
$$

for $k=1,2, \ldots, n$ is the $F$-transform of $f$ in relation to $B_{1}, B_{2}, \ldots, B_{n}$.

Definition 2.5 ([23]) Let $\left[F_{1}, F_{2}, \ldots, F_{n}\right]$ be the $F$-transform of a function $f$ featuring BFs $B_{1}, B_{2}, \ldots, B_{n}$. Then

$$
f_{n}(t)=\sum_{k=1}^{n} F_{k} B_{k}(t)
$$

is called the inverse $F$-transform of a function $f$ on $[a, b]$.

Theorem 2.6 ([23]) Let $B_{1}, B_{2}, \ldots, B_{n}$ be the BFs forming a fuzzy partition of $[a, b]$, and let $f$ be a continuous function on $[a, b]$. Thus, the kth component of the integral F-transform over $[f(a), f(b)]$ gives the minimum of the function

$$
\phi(y)=\int_{a}^{b}(f(t)-y)^{2} B_{k}(t) d t .
$$

Lemma 2.7 ([23] (Convergence)) Let $f$ be a continuous function on $[a, b]$. Thus, for every $\epsilon>0$, there exist $n_{\epsilon}$ and a fuzzy partition $B_{1}, \ldots, B_{n_{\epsilon}}$ of $[a, b]$ such that

$$
\left|f(t)-f_{n_{\epsilon}}(t)\right| \leq \epsilon
$$

for all $t \in[a, b]$. 
Theorem 2.8 Let $B_{1}, B_{2}, \ldots, B_{n}$ be BFs, and let

$$
F T\left(B_{1}, B_{2}, \ldots, B_{n}\right)=\left\{g(t) \mid g(t)=\sum_{i=1}^{n} c_{i} B_{i}(t)\right\}
$$

where $c_{1}, c_{2}, \ldots, c_{n}$ are optional real numbers, and $f \in L_{2}\left([a, b], B_{1}, B_{2}, \ldots, B_{n}\right)$. Considering

$$
\|f\|_{k}=\sqrt{\int_{t_{k-1}}^{t_{k+1}} f^{2}(t) B_{k}(t) d t}, \quad k=1, \ldots, n
$$

and the metric distance $d_{k}(f, g)=\|f-g\|_{k}$, the components $F_{1}, F_{2}, \ldots, F_{n}$ of the F-transform off minimize the sum $\sum_{k=1}^{n} d_{k}^{2}\left(f, c_{k}\right)$ with respect to the parameters $c_{1}, c_{2}, \ldots, c_{n}$.

Proof $L_{2}\left([a, b], B_{1}, B_{2}, \ldots, B_{n}\right)$ is a normed space (see Lemma 5 in [23]). With the metric distance $d_{k}(f, g)$ and Theorem from [23], the proof is complete.

\section{Description of the new approach}

By Theorem 2.8, if $u \in L_{2}\left([0, T], B_{0}, B_{1}, \ldots, B_{n}\right)$, then we get an approximation of the function $u$ for (1.2) with basic functions BFs of the form

$$
u(t) \approx u_{\mathrm{BFT}}(t)=\sum_{k=0}^{n} u_{k} B_{k}(t), \quad t \in[0, T]
$$

where $u_{k}=u\left(t_{k}\right)$ for $k=0,1,2, \ldots, n$ with $t_{k}=k h$, and $h=\frac{T}{n}$ is the time step length (in which $n \geq 1$ is integer). It is evident that $u\left(t_{k}\right)=u_{k}=u_{\mathrm{BFT}}\left(t_{k}\right)$.

Using the approximation for the Caputo derivative [28] of equation (1.3), we have:

$$
D^{\beta} u\left(t_{k+1}\right) \approx \frac{1}{\Gamma(2-\beta) h^{\beta}} \sum_{j=0}^{k}\left(u\left(t_{j+1}\right)-u\left(t_{j}\right)\right)\left((k-j+1)^{1-\beta}-(k-j)^{1-\beta}\right) .
$$

Remark 3.1 Substituting $u_{\mathrm{BFT}}(t)$ into (3.2), we get the equation

$$
D^{\beta} u_{\mathrm{BFT}}\left(t_{k+1}\right) \approx \frac{1}{\Gamma(2-\beta) h^{\beta}} \sum_{j=0}^{k}\left(u_{j+1}-u_{j}\right)\left((k-j+1)^{1-\beta}-(k-j)^{1-\beta}\right) .
$$

Now, to gain the approximate solution of problem (1.2), we use $u_{\mathrm{BFT}}(t)$, and then

$$
\begin{aligned}
& D_{t}^{\beta} u_{\mathrm{BFT}}(t)-\frac{1}{\lambda}\left(u_{\mathrm{BFT}}(t)-u_{\mathrm{BFT}}^{2}(t)-u_{\mathrm{BFT}}(t) \int_{0}^{t} u_{\mathrm{BFT}}(x) d x\right)=0, \\
& 0<\beta \leq 1,0<t \leq T
\end{aligned}
$$

and also

$$
\begin{aligned}
& D^{\beta} u_{\mathrm{BFT}}\left(t_{k}\right)-\frac{1}{\lambda}\left(u_{\mathrm{BFT}}\left(t_{k}\right)-u_{\mathrm{BFT}}^{2}\left(t_{k}\right)-u_{\mathrm{BFT}}\left(t_{k}\right) \int_{0}^{t_{k}} u_{\mathrm{BFT}}(x) d x\right), \\
& \quad k=0,1, \ldots, n
\end{aligned}
$$




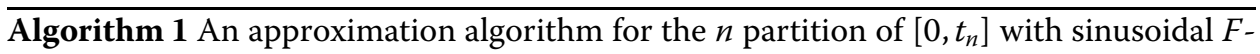
transform.

Step 1. Input $u_{0}=u(0), n$, and $h$.

Step 2. Locate $t_{k} \leftarrow k h, k=0,1,2, \ldots, n$.

Step 3. Choose sinusoidal $B F s B_{k}(t)$ for $k=0,1,2, \ldots, n$.

Step 4. Set

$$
\begin{aligned}
& \frac{1}{h^{\beta} \Gamma(2-\beta)} \sum_{j=0}^{k}\left(u_{j+1}-u_{j}\right)\left((k-j+1)^{1-\beta}-(k-j)^{1-\beta}\right) \\
& \quad-\frac{1}{\lambda}\left(u_{k+1}-u_{k+1}^{2}-\sum_{i=1}^{k+1} a_{i} u_{i} u_{k+1}\right)=0 .
\end{aligned}
$$

Step 5. Calculate every $u_{k}, k=1,2, \ldots, n$, of an equation of degree two.

Step 6. The approximate solution is

$$
u_{\mathrm{BFT}}(t) \approx \sum_{i=0}^{n} u_{i} B_{i}(t)
$$

Regarding (1.3), (3.1), and (3.3), we obtain the following result:

$$
\begin{aligned}
& \frac{1}{h^{\beta} \Gamma(2-\beta)} \sum_{j=0}^{k}\left(u_{j+1}-u_{j}\right) \times\left((k-j+1)^{1-\beta}-(k-j)^{1-\beta}\right) \\
& \quad \approx \frac{1}{\lambda}\left(u_{k+1}-u_{k+1}^{2}-\sum_{i=1}^{k+1} a_{i} u_{i} u_{k+1}\right)
\end{aligned}
$$

where $k=0,1,2, \ldots, n-1, a_{1}=a_{k}=\frac{h}{2}$, and $a_{2}=a_{3}=\cdots=a_{k-1}=h$.

Using the boundary condition $u_{0}=u(0)$, we can calculate $u_{1}, u_{2}, \ldots, u_{n}$ and then get the approximate solution $u(t) \approx u_{\mathrm{BFT}}(t)$ for (1.3).

An approximation for this method stated in Algorithm 1.

Figure 1 shows fractional Volterra population graph obtained by FTM with $n=500$, $\beta=1$, different values of $\lambda=(0.02,0.04,0.1,0.2,0.5), h=0.01$, and $u(0)=0.1$.

\section{Numerical results}

Table 1 for $\beta=1$ illustrates a comparison of the FTM and approximate methods: new homotopic perturbation (NHPM), second derivative multistep approaches (SDMM), rational Chebyshev collocation method (RCC), Hermite functions collocation (HFC), homotopy perturbation method (HPM), with the exact values of [17]

$$
u_{\text {maximum }}=1+\lambda \ln \left(\frac{\lambda}{1+\lambda-u(0)}\right) .
$$

In the last two columns of Table 1 , we can see that by increasing the amount $n$ a more accurate answer can be achieved. 


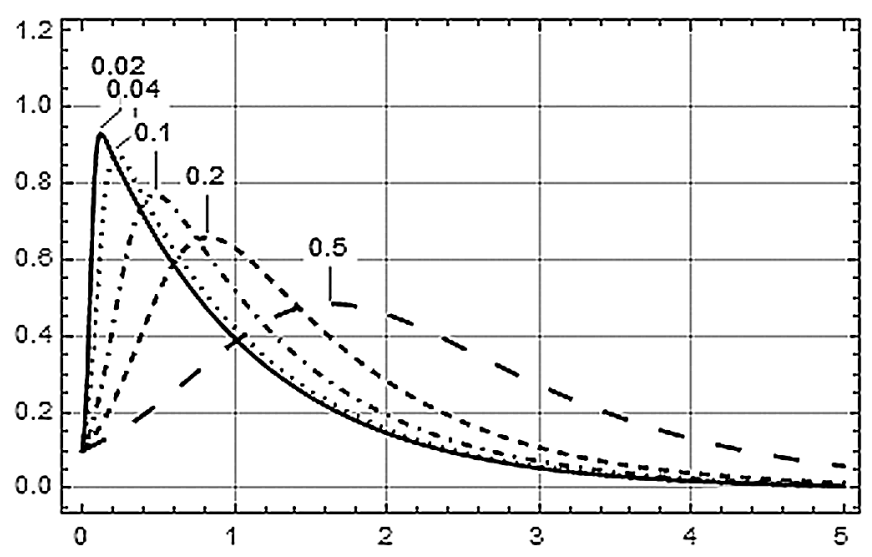

Figure 1 Fractional Volterra population graph obtained by FTM for $\beta=1$.

Table 1 A comparison for $\beta=1$ of the FTM and approximate methods

\begin{tabular}{|c|c|c|c|c|c|c|c|c|}
\hline \multirow[t]{2}{*}{$\lambda$} & \multirow[t]{2}{*}{ NHPM } & \multirow[t]{2}{*}{ HPM } & \multirow[t]{2}{*}{ HFC } & \multirow[t]{2}{*}{$\mathrm{RCC}$} & \multirow[t]{2}{*}{ SDMM } & \multirow[t]{2}{*}{$u_{\max }$} & \multicolumn{2}{|c|}{$\begin{array}{l}\text { Fuzzy transform } \\
\text { method }\end{array}$} \\
\hline & & & & & & & $\begin{array}{l}h=0.01, \\
n=500\end{array}$ & $\begin{array}{l}h=0.001 \\
n=5000\end{array}$ \\
\hline 0.02 & 0.922942037 & 0.90383805 & 0.92342704 & 0.92342715 & 0.92342714 & 0.92342717 & 0.910786 & 0.923059 \\
\hline 0.04 & 0.873725344 & 0.86124017 & 0.87371998 & 0.87371998 & 0.87371998 & 0.87371998 & 0.863645 & 0.873381 \\
\hline 0.10 & 0.765113089 & 0.76511308 & 0.76974149 & 0.76974149 & 0.76974140 & 0.76974149 & 0.760485 & 0.769751 \\
\hline 0.20 & 0.659050432 & 0.65791230 & 0.65905038 & 0.65905038 & 0.65905037 & 0.65905038 & 0.651225 & 0.659057 \\
\hline 0.50 & 0.485190290 & 0.48528234 & 0.48519030 & 0.48519030 & 0.48519029 & 0.48519030 & 0.480624 & 0.485189 \\
\hline
\end{tabular}

\section{Conclusion}

In the present paper, we have applied the sinusoidal BFs for an approximate solution of FVPG. The advantage of this method can be its simple way with approximate accuracy indicated through a given example. As it has been illustrated in the previous section, the approximate solution resulted in this paper is consistent with either exact solution or with other counterparts. Eventually, it is recommended to utilize the proposed approach to solve differential equations, partial integro-differential equations, and equation systems of arbitrary order.

Competing interests

The authors declare that they have no competing interests.

Authors' contributions

All authors read and approved the final manuscript.

\section{Author details}

${ }^{1}$ Department of Mathematics, Çankaya University, Ankara, Turkey. ${ }^{2}$ Institute of Space Sciences, Maturely-Bucharest, Romania. ${ }^{3}$ Department of Mathematics, Qaemshahr Branch, Islamic Azad University, Qaemshahr, Iran. ${ }^{4}$ Department of Mathematics, King Saud University, Riyadh, 11495, Saudi Arabia.

\section{Publisher's Note}

Springer Nature remains neutral with regard to jurisdictional claims in published maps and institutional affiliations.

Received: 3 August 2017 Accepted: 7 November 2017 Published online: 25 November 2017

\section{References}

1. Magin, RL, Abdullah, O, Baleanu, D, Zhou, XJ: Anomalous diffusion expressed through fractional order differential operators in the Bloch-Torrey equation. J. Magn. Res. 190(2), 255-270 (2008) 
2. Scalas, E: The application of continuous-time random walks in finance and economics. Physica A 362(2), 225-239 (2006)

3. Deshpande, AS, Daftardar-Gejji, V, Sukale, YV: On Hopf bifurcation in fractional dynamical systems. Chaos Solitons Fractals 98, 189-198 (2017)

4. Neamaty, A, Nategh, M, Agheli, B: Local non-integer order dynamic problems on time scales revisited. Int. J. Dyn. Control (2017). doi:10.1007/s40435-017-0322-x

5. Raja, MAZ, Samar, R, Alaidarous, ES, Shivanian, E: Bio-inspired computing platform for reliable solution of Bratu-type equations arising in the modeling of electrically conducting solids. Appl. Math. Model. 40(11), 5964-5977 (2016)

6. Guner, O, Bekir, A: The exp-function method for solving nonlinear space-time fractional differential equations in mathematical physics. J. Assoc. Arab Univ. Basic Appl. Sci. 24, 277-282 (2017)

7. Neamaty, A, Nategh, M, Agheli, B: Time-space fractional Burger's equation on time scales. J. Comput. Nonlinear Dyn. 12(3), $031022(2017)$

8. Ming, C, Liu, F, Zheng, L, Turner, I, Anh, V: Analytical solutions of multi-term time fractional differential equations and application to unsteady flows of generalized viscoelastic fluid. Comput. Math. Appl. 72(9), 2084-2097 (2016)

9. Baleanu, D, Luo, AC: Machado, JT (eds.): Discontinuity and Complexity in Nonlinear Physical Systems. Springer, Berlin (2014)

10. Kilbas, AA, Srivastava, HM, Trujillo, JJ: Theory and Application of Fractional Differential Equations. Elsevier, Amsterdam (2006)

11. Podlubny, I: Fractional Differential Equations: An Introduction to Fractional Derivatives, Fractional Differential Equations, to Methods of Their Solution and Some of Their Applications. Mathematics in Science and Engineering, vol. 198. Academic Press, San Diego (1998)

12. Small, RD: Population growth in a closed system. SIAM Rev. 25, 93-95 (1983)

13. Hicdurmaz, B, Can, E: On the numerical solution of a fractional population growth model. Tbil. Math. J. 10(1), 269-278 (2017)

14. Parand, K, Delkhosh, M: Solving Volterra's population growth model of arbitrary order using the generalized fractional order of the Chebyshev functions. Ric. Mat. 65(1), 307-328 (2016)

15. Maleki, M, Kajani, MT: Numerical approximations for Volterra's population growth model with fractional order via a multi-domain pseudospectral method. Appl. Math. Model. 39(15), 4300-4308 (2015)

16. Suat Erturk, V, Yildirım, A, Momanic, S, Khan, Y: The differential transform method and Padé approximants for a fractional population growth model. Int. J. Numer. Methods Heat Fluid Flow 22(6), 791-802 (2012)

17. Khan, NA, Ara, A, Jamil, M: Approximations of the nonlinear Volterra's population model by an efficient numerical method. Math. Methods Appl. Sci. 34(14), 1733-1738 (2011)

18. Krishnaveni, K, Kannan, K, Balachandar, SR: Approximate analytical solution for fractional population growth model. Int. J. Eng. Technol. 5(3), 2832-2836 (2013)

19. Dehghan, M, Shahini, M: Rational pseudospectral approximation to the solution of a nonlinear integro-differentia equation arising in modeling of the population growth. Appl. Math. Model. 39(18), 5521-5530 (2015)

20. Ghasemi, M, Fardi, M, Ghaziani, RK: A new application of the homotopy analysis method in solving the fractional Volterra's population system. Appl. Math. 59(3), 319-330 (2014)

21. Yildirim, A, Gulkanat, Y: HPM-Pade technique for solving a fractional population growth model. World Appl. Sci. J. 11(12), 1528-1533 (2010)

22. Fathizadeh, E, Ezzati, R, Maleknejad, K: Hybrid rational Haar wavelet and block pulse functions method for solving population growth model and Abel integral equations. Math. Probl. Eng. 2017, 2465158 (2017)

23. Perfilieva, I: Fuzzy transforms: theory and applications. Fuzzy Sets Syst. 157(8), 993-1023 (2006)

24. Khastan, A, Perfilieva, I, Alijani, Z: A new fuzzy approximation method to Cauchy problems by fuzzy transform. Fuzzy Sets Syst. 288, 75-95 (2016)

25. Khastan, A, Alijani, Z, Perfilieva, I: Fuzzy transform to approximate solution of two-point boundary value problems. Math. Methods Appl. Sci. 40, 6147-6154 (2017)

26. Tomasiello, S: An alternative use of fuzzy transform with application to a class of delay differential equations. Int. J. Comput. Math. 94, 1719-1726 (2017)

27. Chen, W, Shen, Y: Approximate solution for a class of second-order ordinary differential equations by the fuzzy transform. J. Intell. Fuzzy Syst. 27(1), 73-82 (2014)

28. Li, C, Zhao, Z, Chen, Y: Numerical approximation of nonnlinear fractional differential equations with subdiffusion and superdiffusion. Comput. Math. Appl. 62(3), 855-875 (2011)

\section{Submit your manuscript to a SpringerOpen ${ }^{\circ}$ journal and benefit from:}

- Convenient online submission

- Rigorous peer review

- Open access: articles freely available online

- High visibility within the field

- Retaining the copyright to your article

Submit your next manuscript at $\boldsymbol{~ s p r i n g e r o p e n . c o m ~}$ 